\title{
Utility of endoscopic ultrasound-guided fine-needle biopsy in the diagnosis of type 1 autoimmune pancreatitis*
}

\section{다)(우우}

\author{
Authors \\ Beate Haugk ${ }^{3}$ \\ Institutions \\ 1 HPB Unit, Freeman Hospital, Newcastle upon Tyne \\ Hospitals NHS Foundation Trust, Newcastle upon Tyne, \\ United Kingdom \\ 2 Translational and Clinical Research Institute, Newcastle \\ University, Newcastle upon Tyne, United Kingdom \\ 3 Department of Cellular Pathology, Royal Victoria \\ Infirmary, Newcastle upon Tyne Hospitals NHS \\ Foundation Trust, Newcastle upon Tyne, United \\ Kingdom \\ 4 Population Health Sciences Institute, Newcastle \\ University, Newcastle Upon Tyne, United Kingdom
}

Kofi W. Oppong1,2, Pardeep Maheshwari ${ }^{1}$, Manu K. Nayar ${ }^{1}$, Antony Darne ${ }^{3}$, Daniel Parkinson ${ }^{3}$, John S. Leeds ${ }^{1,4}$,

submitted 28.5.2010

accepted after revision 23.7.2020

Bibliography

Endoscopy International Open 2020; 08: E1855-E1861

DOI 10.1055/a-1236-3266

ISSN 2364-3722

(C) 2020. The Author(s)

This is an open access article published by Thieme under the terms of the Creative Commons Attribution-NonDerivative-NonCommercial License, permitting copying and reproduction so long as the original work is given appropriate credit. Contents may not be used for commecial purposes, or adapted, remixed, transformed or built upon. (https://creativecommons.org/licenses/by-nc-nd/4.0/)

Corresponding author

Dr. K.W. Oppong, Consultant Gastroenterologist, HPB Unit Freeman Hospital, Newcastle upon Tyne, NE7 7DN, UK

Fax: +00441912231249

Kofi.oppong@nhs.net

\section{ABSTRACT}

Background and study aims Endoscopic ultrasound (EUS)-guided fine-needle aspiration (FNA) performs poorly in the histological diagnosis of type 1 autoimmune pancreatitis (AIP). The aim of this study was to assess the performance of fine-needle biopsy (FNB) comparing reverse bevel (RB) and fork-tip (FT) needles.

Patients and methods A retrospective study of prospectively maintained databases was performed. Patients with a final diagnosis of type 1 AIP who underwent EUS-FNB during diagnostic workup were included. Pathology reports were reviewed and classified as per international consensus diagnostic criteria (ICDC). The Primary outcome was EUSFNB sensitivity in diagnosing type 1 AIP.

Results Between March 2011 and December 2018, 24 patients with a final diagnosis of type 1 AIP underwent FNB. Six patients underwent biopsy with the RB needle and 18 with the FT needle. Mean age $( \pm$ SD) $62.2( \pm 11.4), 17$ (70.8\%) male. No RB samples were diagnostic compared to 14 (78\%) FT; $P=0.001$; of which 13 (72\%) were level 1. In eight $(44 \%)$ of FT cases a diagnosis was not possible without histology. Initial biopsy was diagnostic in five (62.5\%) of these cases. Including repeat biopsy, seven (87\%) had a diagnosis made by FT needle. Obliterative phlebitis (44\%) was the least frequently identified pathological feature and immunoglobulin ( $\mathrm{lgG}) 4$ + plasma cells $>10$ per high power field $(78 \%)$ the most common.

Conclusion The FT needle demonstrated good performance for diagnosing type 1 AIP. The results support the preferential use of this core biopsy needle for EUS pancreatic tissue sampling.

\section{Introduction}

IgG4-related disease (IgG4-RD) is a rare immune mediated fibroinflammatory condition that can affect nearly any organ; it manifests in the pancreas as type 1 autoimmune pancreatitis

\footnotetext{
* Meeting presentations: Pancreatic Society of Great Britain and Ireland November 2018, Digestive Disease Week May 2019, and British Society of Gastroenterology annual meeting June 2019
}

(AIP). A number of criteria have been developed for its diagnosis, including the International consensus criteria (ICDC) [1] and the modified HISORt (Histology, Imaging, Serology, Other organ involvement, and Response to therapy) criteria [2]. AIP commonly presents similarly to pancreatic ductal adenocarcinoma (PDAC) with painless obstructive jaundice, differentiation from PDAC is therefore crucial.

Histology is regarded as the gold standard for the diagnosis of IgG4-RD irrespective of the organ involved [3]. The three de- 


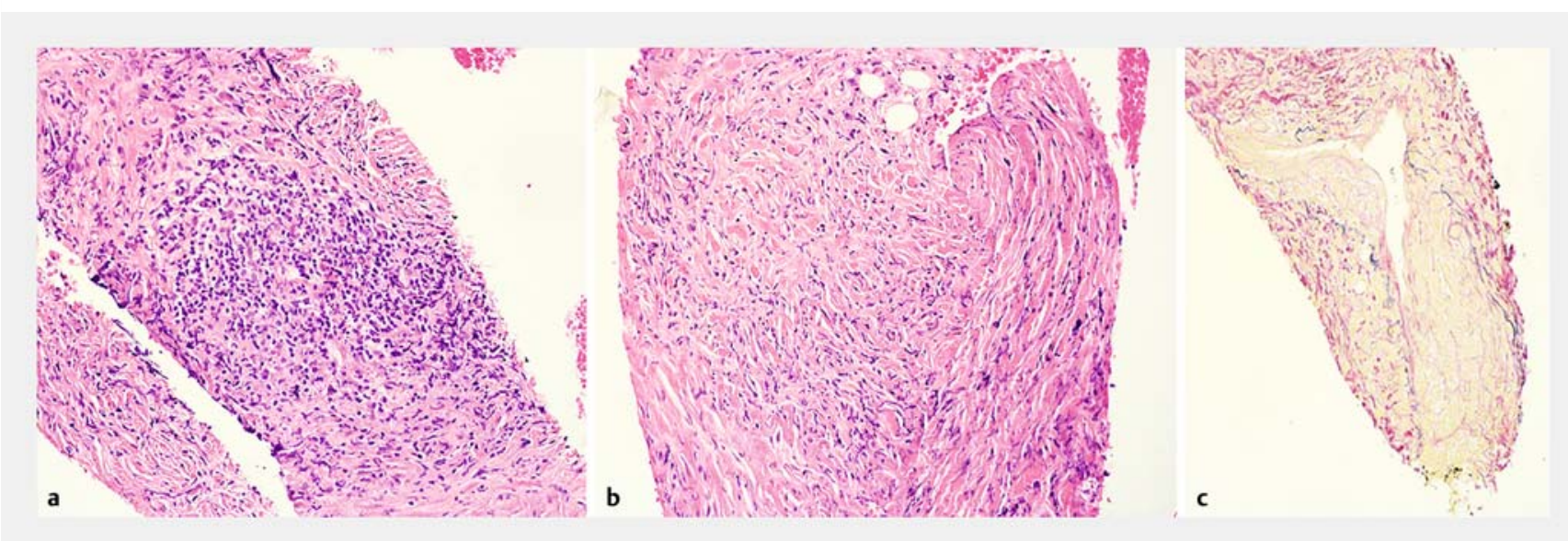

- Fig. 1 Characteristic histological features of Type 1 AIP seen on fork-tip biopsy sample. a Lymphoplasmacytic infiltration (H\&E staining). b Storiform fibrosis(H\&E staining). c Obliterative phlebitis (elastic van Gieson staining).

fining morphological features comprise dense lymphoplasmacytic inflammation, storiform fibrosis i.e. fibrosis arranged at least focally in a storiform pattern, and obliterative phlebitis. Additional immunohistochemical evidence is provided by a tissue specific elevated number of IgG4 positive plasma cells per high power field $\left(\mathrm{IgG} 4^{+} /\right.$high-power field [HPF]) and a greater than $40 \%$ ratio of Ig4 to IgG-positive plasma cells [4,5]. EUSguided tissue sampling (historically fine-needle aspiration [FNA] cytology) is the standard of care for diagnosing pancreatic malignancy with sensitivity of $\approx 85 \%$ [6]. However, the majority of studies of EUS-FNA in AIP using standard techniques have documented poor diagnostic performance as the diagnosis of AIP requires intact tissue $[7,8]$

EUS fine-needle biopsy (EUS-FNB) with acquisition of tissue cores for histological assessment offers the potential benefit of preserved tissue architecture and therefore the possibility of diagnosing AIP on EUS biopsies. The reverse bevel (RB) needle (ProCore, Cook Ireland, Limerick, Ireland), was the first widely adopted core biopsy needle; more recently second-generation core biopsy needles have been introduced including the fork-tip (FT) needle (Sharkcore, Medtronic, Whiteley, UK).

The aim of our study was to assess the diagnostic performance of FNB utilizing RB and FT needles in the diagnosis of type 1 AIP.

\section{Patients and methods}

The primary outcome measure was sensitivity of fork-tip (FT) core biopsy needle and reverse bevel (RB) needle in diagnosing type 1 AIP among patients with a final diagnosis of type 1 AIP. Secondary outcome measures were sensitivity of FT core biopsy needle and RB needle in diagnosing type 1 AIP among patients with a final diagnosis of type 1 AIP in whom a definitive diagnosis of type 1 AIP was not possible without histology and evaluation of the frequency of identification of the individual ICDC pathological features of type 1 AIP.

A retrospective review of prospectively maintained AIP and EUS databases in our tertiary center was performed. Eligible pa-

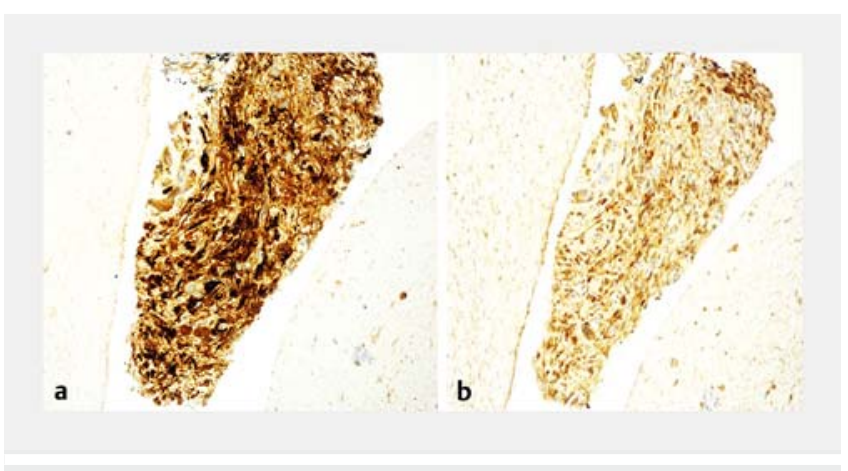

- Fig. 2 Characteristic immunohistochemistry of Type 1 AIP seen on fork-tip biopsy sample. a IgG4 ${ }^{+}$plasma cell infiltration (>10/hpf). b lgG $4^{+}$cells to $\operatorname{lgG}{ }^{+}$cell ratio greater than $40 \%$.

tients were those with a final diagnosis of type 1 AIP who underwent EUS-FNB during diagnostic workup. The EUS database recorded the putative diagnosis based on EUS morphology, the needle type and gauge used for FNB as well as the number of passes. All patients underwent triple phase pancreas protocol computerized tomography (CT) scan prior to EUS-FNB. For the purpose of the study CT scan reports were classified into one of three categories: 1) mass lesion; 2) diffuse enlargement of the pancreas; and 3) stricture of the intra-pancreatic bile duct without visible mass. Serum IgG4 measured shortly before or after EUS-FNB was recorded and expressed relative to the upper limit of normal of the assay used. Standard histopathology reports issued at the time were independently reviewed by a gastroenterologist (KO) and pathologist ( $\mathrm{BH})$. In addition, the slides were reviewed by an experienced pancreatic pathologist $(\mathrm{BH})$ to confirm the presence of the originally reported histological features. The histological ( Fig.1) and immunohistochemical ( $\triangleright$ Fig.2) features of type 1 AIP as defined by ICDC criteria [1] were then collated. Non-obliterative phlebitis and presence of eosinophils were reported as additional features when present.

Cases were then classified as per ICDC criteria [1]. Level 1 (highly suggestive) diagnosis requires $\geq 3$ criteria from among 
lymphoplasmacytic inflammation, storiform fibrosis, obliterative phlebitis and abundant (> $10 \mathrm{lgG} 4^{+} / \mathrm{HPF}$ ) and level 2 (probable) requires two criteria. Final gold standard diagnosis was based on the modified HISORt criteria [2]. There was a minimum of 12 month follow up from the time of diagnosis.

\section{EUS-FNB procedure}

All procedures were performed under conscious sedation. The endoscopist was not blinded to the results of prior investigations. Pentax linear echo-endoscopes (Pentax, Slough, UK) and Hitachi ultrasound workstations (Hitachi Medical Systems, Wellingborough, UK) were used. FNB has been the EUS sampling method of choice in our unit since 2011. The slow pull technique was used for some procedures with a 25-gauge needle, suction was otherwise used, and the fanning technique used for all procedures. The needle was moved backward and forward within the lesion for six to 10 throws. Suction was stopped prior to withdrawal of the needle. A minimum of three passes were performed unless precluded by patient or lesion factors. All samples from a procedure were placed in a single container of $10 \%$ neutral buffered formalin and fixed for a minimum of 4 hours.

\section{Histological processing and reporting}

Samples were subsequently processed on a Leica Peloris processor and embedded in paraffin on Leica arcadia embedding centers using Cellwax Plus made by CellPath. A single 4-micron section was cut using Leica RM2245 microtomes and stained with hematoxylin and eosin (H\&E). Serial H\&E sections, elastic van Gieson as per local protocol, anti-IgG4 antibody (mouse anti-human monoclonal antibody, dilution 1:150, The Binding Site Ltd, Birmingham, UK) and anti-IgG antibody (polyclonal rabbit anti-human antibody, dilution 1:60,000, Dakopatts, Glostrup, Denmark) were used as required.

Histological evaluation was performed as per above defined criteria. All slides were double reported. The pathologists were not blinded to prior investigations.

\section{Statistical analysis}

Continuous variables were reported as mean and standard deviation if normally distributed and the median and inter-quartile range otherwise. Continuous variables were compared using the independent samples T test or the Mann-Whitney as appropriate. Categorical data were compared using Fishers exact test and Cochran's Q test. Statistical analysis was performed using MedCalc Statistical Software version 19.2 (MedCalc Software Ltd, Ostend, Belgium; https://www.medcalc.org; 2020).

\section{Ethics approval}

The study was performed as a service evaluation using routinely collected clinical data and in accordance with United Kingdom National Health Service research ethics guidance ethical approval from an institutional review body was not required for this study. Institutional authorization to hold a prospective database for service evaluation was obtained. Written informed consent was obtained from all patients prior to the procedure.

\section{Results}

There was no discrepancy in endoscopist and pathologist classification of the pathology reports and there was no change in classification following pathologist review of the slides. There were 28 EUS-FNB procedures in 24 individuals between March 2011 and December 2018. The RB needle was the preferred needle for EUS tissue sampling in the initial period and the FT latterly. Six individuals underwent six procedures with the RB needle and 18 individuals underwent 21 procedures with the FT (> Fig. 3).

Mean age, (SD) was 62.2 years (11.4), 17 (71\%) were male. Nineteen individuals (79\%) had biliary obstruction at the time of presentation and serum IgG4 was elevated in 16 (69\%). There was no significant difference in any baseline ( $\downarrow$ Table 1 ) or EUS procedure ( $\downarrow$ Table 2 ) characteristic between the FT needle biopsy group and the RB needle biopsy group.

The pathological findings and ICDC histological diagnostic level for the index FNB procedure are shown in $>$ Table 3 . An adequate specimen was obtained in four patients $(67 \%)$ in the RB group compared to $17(94 \%)$ in the FT group $(P=0.14)$. A histological diagnosis of type 1 AIP was not made on any RB needle biopsy compared to $14(78 \%)$ with the FT needle $(P=$ $0.001) ; 13$ of the diagnostic samples were at level 1. Regarding the histological and immunohistochemical features of IgG4 ( $\triangleright$ Table 3) an IgG4 ${ }^{+}$plasma cell infiltrate (>10/hpf) was the most commonly seen feature, being identified in $78 \%$ of cases and obliterative phlebitis the least (seen in 44\%) however there was no significant difference among the proportions $(P=0.06)$.

Of the 10 patients with non-diagnostic histology, seven (6 $\mathrm{RB}$ and $1 \mathrm{FT}$ group) had a definitive diagnosis made without histology based on other HISORt criteria ( $>$ Fig.3). Two were based on other features (1 FT and 1 RB case) and five (RB cases) on response to steroids. The three non-diagnostic FT cases in whom a definitive diagnosis was not possible without histology underwent repeat FT biopsy of which two were diagnostic (level 1). The cumulative diagnostic rate with the FT needle was therefore 16 of 18 (89\%). Of the eight FT cases in whom a diagnosis of type 1 AIP was not possible without histology five $(62.5 \%)$ had a diagnosis made on initial biopsy, including repeat biopsy the number was seven (87.5\%).

Two patients in the FT group were submitted to surgery. The first, a 54-year-old male with a head of pancreas mass causing double duct dilatation and a CT and EUS appearance in keeping with pancreatic malignancy (serum IgG4 not measured), underwent fast-track pancreaticoduodenectomy before the diagnostic (level 2) FT biopsy histology report was available. The second, a 62-year-old female with biliary obstruction secondary to a mass in the head of pancreas with normal serum IgG4 and elevated CA 19/9, underwent laparoscopic biopsy and hepaticojejunostomy for suspected malignancy following two non-diagnostic biopsies with the FT needle. 


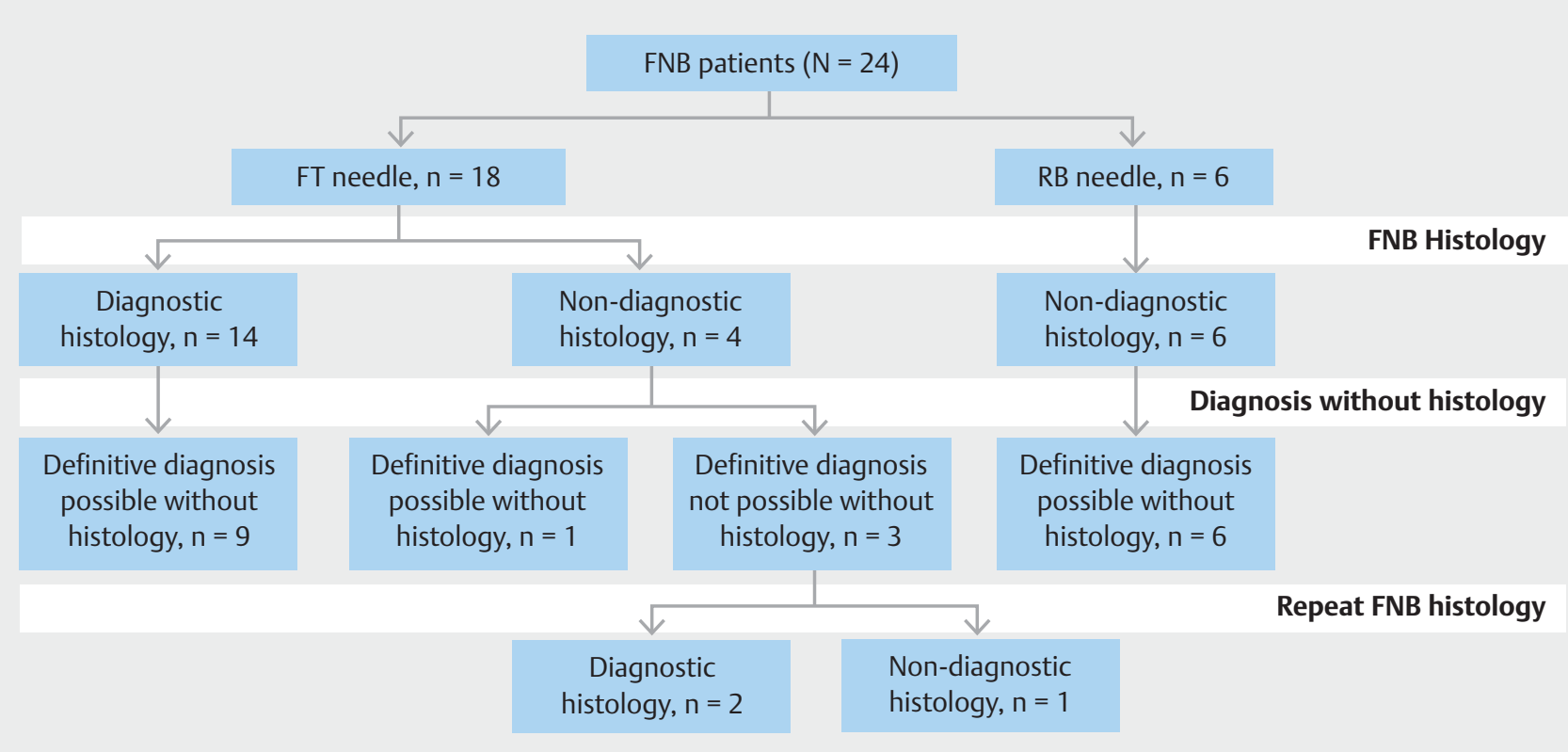

- Fig.3 FNB needle group allocation, diagnostic yield, and cases in which a diagnosis by HISORt criteria was possible without histology.

- Table 1 Baseline characteristics.

\begin{tabular}{|c|c|c|c|c|}
\hline Characteristics & All patients $(n=24)$ & Fork-tip needle $(n=18)$ & Reverse bevel needle ( $n=6$ ) & $P$ value \\
\hline Male sex, n (\%) & $17(71 \%)$ & $11(61 \%)$ & $6(100 \%)$ & 0.13 \\
\hline Age, years, mean \pm SD (range) & $62.2 \pm 11.4(41-82)$ & $63.3 \pm 10.14(41-77)$ & $59.2 \pm 15.2(42-82)$ & 0.45 \\
\hline \multicolumn{4}{|l|}{ Imaging } & 0.82 \\
\hline - Diffuse pancreatic enlargement & $1354 \%)$ & $9(50 \%)$ & $4(67 \%)$ & \\
\hline - Mass & $8(33 \%)$ & $7(39 \%)$ & $1(17 \%)$ & \\
\hline - Biliary stricture & $3(12 \%)$ & $2(11 \%)$ & $1(17 \%)$ & \\
\hline Biliary obstruction, n (\%) & $19(79 \%)$ & $14(78 \%)$ & $5(83.3 \%)$ & 1.00 \\
\hline \multicolumn{4}{|l|}{ Serum $\lg G 4, \mathrm{n}(\%)^{1}$} & 0.26 \\
\hline - <upper limit of normal, n (\%) & $7(30 \%)$ & $4(23 \%)$ & $3(50 \%)$ & \\
\hline - 1-2 × upper limit of normal, n (\%) & $5(22 \%)$ & $3(18 \%)$ & $2(33 \%)$ & \\
\hline - >2 × upper limit of normal, $\mathrm{n}(\%)$ & $11(48 \%)$ & $10(59 \%)$ & $1(17 \%)$ & \\
\hline
\end{tabular}

\section{Discussion}

Histology is a key criterion for the diagnosis of AIP [1,9]. EUS tissue sampling is commonly performed in the investigation of suspected pancreatic malignancy. However current guidelines do not specify a role for EUS or EUS tissue sampling in the diagnosis of type 1 AIP beyond the exclusion of malignancy [9] in patients presenting with a focal mass or biliary stricture. In the present study the RB needle did not provide a diagnostic sample whereas sensitivity with the FT needle was $78 \%$; $P=0.001$. The sample size of this retrospective study was small, reflecting the rarity of the disease and the fact that not all patients undergo EUS tissue sampling.

The likely reason for the superior performance of the FT needle in diagnosing type 1 AIP is its greater likelihood of acquiring intact tissue cores compared to the RB needle [10], thereby allowing better assessment of architectural features including pattern of stroma and the relation of stroma and inflammation. Veins may also be more readily identified if the tissue is intact.

A significant proportion of cases in the present study had features concerning for malignancy with biliary obstruction in $79 \%$ and a focal mass in $33 \%$. Without a diagnostic biopsy, a greater proportion would likely have undergone surgery be- 
- Table 2 Characteristics of EUS procedures.

\begin{tabular}{|c|c|c|c|c|}
\hline EUS Characteristics & All procedures $(n=24)$ & Fork-tip needle $(n=18)$ & Reverse bevel needle $(n=6)$ & $P$ value \\
\hline \multicolumn{5}{|c|}{ Suspected diagnosis on EUS appearance } \\
\hline - Autoimmune pancreatitis, n (\%) & $15(57 \%)$ & $11(61 \%)$ & $4(67 \%)$ & \\
\hline \multicolumn{4}{|l|}{ Needle gauge } & 0.28 \\
\hline . $\geq 22 \mathrm{~g}^{1}$ & 19 & 13 & 6 & \\
\hline $.25 \mathrm{~g}$ & 5 & 5 & 0 & \\
\hline Mean number of passes, $\mathrm{n} \pm \mathrm{SD}$ & $2.42 \pm 0.71$ & $2.55 \pm 0.70$ & $2.00 \pm 0.70$ & 0.10 \\
\hline
\end{tabular}

- Table 3 Pathological findings and ICDC histological diagnostic level.

\begin{tabular}{|c|c|c|c|c|}
\hline Pathological attribute & All $(n=24)$ & Fork-tip needle $(n=18)$ & Reverse bevel needle $(n=6)$ & $P$ value \\
\hline Specimen adequacy, n (\%) & $21(87 \%)$ & $17(94 \%)$ & $4(67 \%)$ & 0.14 \\
\hline \multicolumn{5}{|l|}{ Histology } \\
\hline - Storiform fibrosis, n (\%) & $11(46 \%)$ & $11(61 \%)$ & 0 & \\
\hline - Obliterative phlebitis, n(\%) & $8(33 \%)$ & $8(44 \%)$ & 0 & \\
\hline - Lymphoplasmacytic infiltration, n (\%) & $12(50 \%)$ & $12(67 \%)$ & 0 & \\
\hline \multicolumn{5}{|l|}{ Immunohistochemistry } \\
\hline - $\operatorname{lgG} 4+/ \mathrm{HPF}>10, \mathrm{n}(\%)^{1}$ & $1562 \%)$ & $14(78 \%)$ & $1(17 \%)$ & \\
\hline - $\operatorname{lgG} 4+/ \operatorname{lgG}+$ plasma cell ratio $>40 \%, \mathrm{n}(\%)^{2}$ & $7(29 \%)$ & $7(39 \%)$ & 0 & \\
\hline \multicolumn{5}{|l|}{ ICDC diagnostic level } \\
\hline - $0, \mathrm{n}(\%)$ & $10(42 \%)$ & $4(22 \%)$ & 6 & \\
\hline - $1, n(\%)$ & $13(54 \%)$ & $13(72 \%)$ & 0 & \\
\hline - $2, n(\%)$ & $1(4 \%)$ & $1(7 \%)$ & 0 & \\
\hline ICDC diagnosis (level 1 or 2), n (\%) & $14(58 \%)$ & $14(78 \%)$ & 0 & 0.001 \\
\hline
\end{tabular}

cause of concerns over using a trial of steroids to distinguish between type 1 AIP and malignancy [9].

Misdiagnosis of PDAC as AIP can lead to inappropriate steroid therapy and delay in surgery or chemotherapy [11]. On the other hand, misdiagnosis as PDAC can lead to unnecessary surgery. In a recent UK series [12] $60 \%$ of AIP cases underwent initial resection as presumed malignancy. In a large Dutch series, $2.6 \%$ of pancreatoduodenectomies had a final diagnosis of AIP [13]. This data emphasizes the utility of a definitive tissue diagnosis in patients presenting with biliary obstruction and or a pancreatic mass.

EUS-FNA with cytological evaluation of samples has been the preferred EUS-guided tissue sampling technique for the diagnosis of pancreatic masses and has good performance for diagnosing pancreatic malignancy [6]. However, FNA cytology does not provide enough intact tissue to allow a histological diagno- sis of AIP [8]. Furthermore, only the presence of lymphoplasmacytic inflammatory cells could be assessed cytologically, but their density could not be evaluated. While immunohistochemistry for IgG4 can be applied to cytology preparations, it would not be possible to count their proportion in high power fields of intact tissue.

Previous EUS-FNA studies have reported poor to modest diagnostic performance. A prospective, multicenter study evaluating 50 patients with suspected AIP using a 22-gauge FNA needle reported a sensitivity of $7.9 \%$ [7]. Iwashita et al [14] reported an overall accuracy of $43 \%$. More recently utilizing $22 \mathrm{~g}$ FNA needles Kanno et al [15] reported $57.7 \%$ overall accuracy by ICDC criteria with $41 \%$ achieving a level 1 diagnosis. However, the study employed a specialized, laborious technique involving piecing together tissue fragments; this approach cannot readily be adopted in routine practice. In another recent 
study [16] utilizing 22-gauge FNA needles among 27 AIP patients, $18.5 \%$ and $44.4 \%$ cases were assessed as having level 1 and level 2 diagnosis respectively. Obliterative phlebitis was not detected in any samples. These previous studies highlight the difficulties in making a pathological diagnosis of AIP (particularly level 1) from FNA samples even when processed histologically and specialized techniques applied.

EUS-guided Tru-cut (Quickcore, Cook Ireland, Limerick, Ireland) was the first routinely available EUS core biopsy and showed some utility in diagnosis of AIP in small series [17-19]. However, due to technical difficulties in deploying the needle, the device never achieved widespread use.

New core biopsy needles have been developed with the aim of improving tissue quantity and architectural integrity to facilitate histological analysis. The RB needle was the first widely used core biopsy needle; however, this has not shown superiority to FNA in the diagnosis of pancreatic malignancy $[20,21]$. More recently FT and Franseen needles have been introduced, as well as a 20-gauge forward-bevel needle. A recent randomized crossover trial documented significantly better sensitivity of the FT needle in diagnosing pancreatic malignancy compared to FNA [22].

With regard to AIP, there have been a number of case reports [23-25] of its diagnosis using the FT needle. Kurita et al [26] recently published a prospective multicenter, randomized controlled trial of a 22-gauge Franseen needle and a 20 gauge forward-bevel needle among 110 patients with suspected type 1 AIP. Seventy-eight percent of patients in the Franseen group and $45 \%$ in the forward-bevel group were diagnosed with type 1 AIP; a level 1 diagnosis was achieved in $56 \%$ and $26 \%$ of patients, respectively. Obliterative phlebitis was the least readily identified histological feature and was seen in $24 \%$ in the Franseen group and $14 \%$ in the forward-bevel group. The results with the FT needle in our study are comparable to the Franseen needle results reported in the Kurita study, with similar sensitivity and obliterative phlebitis being the least identified histological feature.

The results of the current study add to the evidence that obliterative phlebitis is the most difficult histological feature to identify on small biopsy samples. Possible reasons for this include the paucity of vessels in small samples and the fact that reliably recognizing obliterative phlebitis and distinguishing it from venous scars is challenging.

A ratio of IgG4-positive to IgG-positive cells $>40$ although not one of the ICDC criteria has been suggested as one of the most sensitive features for the diagnosis of IgG4 related disease [5]. However, the evaluation of immunohistochemistry for accurate counts of $\mathrm{IgG} 4$ and $\mathrm{lgG}$ positive cells from small samples can be technically challenging [3], especially in terms of background staining; as was shown in our study.

This study has several limitations including its retrospective design; however, conducting a prospective study in such a rare disease would be challenging. Furthermore, the study design precluded blinding of the pathologists to clinical details and prior results, including the EUS report. However, the diagnosis of AIP was not suspected in a significant proportion at the time of EUS. Additionally, as the study was conducted over several years, with the RB needle used in the early part of the study, it is possible that there was a learning curve effect. However, study endosonographers and pathologists had significant experience of the endosonographic and histological features of AIP respectively prior to the study period.

A strength of the study is the fact that all specimens were double reported by two specialist pancreatic pathologists with expertise in both AIP and EUS-FNB prior to issue of the original clinical report. In addition, the slides were reviewed by an expert pathologist for the purpose of the study.

Another strength of the study is case ascertainment and follow up. Cases were identified from our multispeciality IgG4-RD database, in which all cases with a final diagnosis of type 1 AIP are registered. All cases with a suspicion of type 1 AIP remain under close follow up with further tissue sampling, imaging and trial of steroids as appropriate until type 1 AIP is either confirmed or excluded. We believe therefore that the risk of selection bias is low.

\section{Conclusion}

In conclusion, this study demonstrates very poor performance of the RB needle and good performance of the FT needle in diagnosing type 1 AIP and provides further evidence to support the preferential use of second-generation core biopsy needles in EUS-guided sampling of pancreatic disease and their interpretation by experienced, specialist pathologists.

\section{Competing interests}

Dr. Oppong has received a research grant, travel grant, and speaker fees from Medtronic. Drs. Nayar and Haugk have received research grants and speaker fees from Medtronic. Drs. Leeds and Darne have received research grants from Medtronic.

\section{References}

[1] Shimosegawa T, Chari ST, Frulloni L et al. International consensus diagnostic criteria for autoimmune pancreatitis: guidelines of the International Association of Pancreatology. Pancreas 2011; 40: 352328

[2] Chari ST, Smyrk TC, Levy M] et al. Diagnosis of autoimmune pancreatitis: The Mayo Clinic Experience. Clin Gastroenterol Hepatol 2006; 4: 1010-1016

[3] Arora K, Rivera M, Ting DT et al. The histological diagnosis of IgG4related disease on small biopsies: challenges and pitfalls. Histopathology 2019; 74: 688-698

[4] Detlefsen S, Kloppel G. IgG4-related disease: with emphasis on the biopsy diagnosis of autoimmune pancreatitis and sclerosing cholangitis. Virchows Arch 2018; 472: 545-556

[5] Deshpande V, Zen Y, Chan JKC et al. Consensus statement on the pathology of IgG4-related disease. Mod Pathol 2012; 25: 1181-1192

[6] Puli SR, Bechtold ML, Buxbaum JL et al. How good is endoscopic ultrasound-guided fine-needle aspiration in diagnosing the correct etiology for a solid pancreatic mass? A meta-analysis and systematic review. Pancreas 2013; 42: 20-26 
[7] Morishima T, Kawashima H, Ohno E et al. Prospective multicenter study on the usefulness of EUS-guided FNA biopsy for the diagnosis of autoimmune pancreatitis. Gastrointest Endosc 2016; 84: 241-248

[8] Majumder S, Chari ST. EUS-guided FNA for diagnosing autoimmune pancreatitis: Does it enhance existing consensus criteria? Gastrointest Endosc 2016; 84: 805-807

[9] Nagpal SJS, Sharma A, Chari ST. Autoimmune Pancreatitis. Am J Gastroenterol 2018; 113: 1301

[10] Bang JY, Krall K, Jhala N et al. Comparing needles and methods of endoscopic ultrasound-guided fine-needle biopsy to optimize specimen quality and diagnostic accuracy for patients with pancreatic masses in a randomized trial. Clin Gastroenterol Hepatol 2020: doi:10.1016/j.cgh.2020.06.042

[11] Gardner TB, Levy MJ, Takahashi N et al. Misdiagnosis of autoimmune pancreatitis: a caution to clinicians. Am J Gastroenterol 2009; 104: 1620-1623

[12] Dickerson LD, Farooq A, Bano F et al. Differentiation of Autoimmune pancreatitis from pancreatic cancer remains challenging. World J Surg 2019; 43: 1604-1611

[13] van Heerde MJ, Biermann K, Zondervan PE et al. Prevalence of autoimmune pancreatitis and other benign disorders in pancreatoduodenectomy for presumed malignancy of the pancreatic head. Dig Dis Sci 2012; 57: 2458-2465

[14] Iwashita T, Yasuda I, Doi S et al. Use of samples from endoscopic ultrasound-guided 19-gauge fine-needle aspiration in diagnosis of autoimmune pancreatitis. Clin Gastroenterol Hepatol 2012; 10: 316322

[15] Kanno A, Masamune A, Fujishima F et al. Diagnosis of autoimmune pancreatitis by EUS-guided FNA using a 22-gauge needle: a prospective multicenter study. Gastrointest Endosc 2016; 84: 797-804.e1

[16] Cao L, Wang Y, Wang J et al. The role of EUS-guided fine-needle aspiration in autoimmune pancreatitis: a single center prospective study. Scand J Gastroenterol 2018; 53: 1604-1610
[17] Mizuno N, Bhatia V, Hosoda W et al. Histological diagnosis of autoimmune pancreatitis using EUS-guided trucut biopsy: A comparison study with EUS-FNA. J Gastroenterol 2009; 44: 742-750

[18] Levy MJ, Smyrk TC, Takahashi N et al. Idiopathic duct-centric pancreatitis: Disease description and endoscopic ultrasonography-guided trucut biopsy diagnosis. Pancreatology 2011; 11: 76-80

[19] Levy MJ, Reddy RP, Wiersema MJ et al. EUS-guided trucut biopsy in establishing autoimmune pancreatitis as the cause of obstructive jaundice. Gastrointest Endosc 2005; 61: 467-472

[20] Bang JY, Hawes R, Varadarajulu S. A meta-analysis comparing ProCore and standard fine-needle aspiration needles for endoscopic ultrasound-guided tissue acquisition. Endoscopy 2016; 48: 339-349

[21] Khan MA, Grimm IS, Ali B et al. A meta-analysis of endoscopic ultrasound-fine-needle aspiration compared to endoscopic ultrasoundfine-needle biopsy: diagnostic yield and the value of onsite cytopathological assessment. Endosc Int Open 2017; 5: E363-E375

[22] Oppong KW, Bekkali NLH, Leeds JS et al. FT needle biopsy versus fineneedle aspiration in endoscopic ultrasound-guided sampling of solid pancreatic masses: a randomized crossover study. Endoscopy 2020: doi:10.1055/a-1114-5903

[23] Bhattacharya A, Cruise M, Chahal P. Endoscopic ultrasound guided 22 gauge core needle biopsy for the diagnosis of autoimmune pancreatitis. Pancreatology 2018; 18: 168-169

[24] Runge TM, Hart PA, Sasatomi E et al. Diagnosis of autoimmune pancreatitis using new, flexible EUS core biopsy needles: report of two cases. Gastrointest Endosc 2017; 85: 1311-1312

[25] Detlefsen S, Joergensen MT, Mortensen MB. Microscopic findings in EUS-guided fine-needle (SharkCore) biopsies with type 1 and type 2 autoimmune pancreatitis. Pathol Int 2017; 67: 514-520

[26] Kurita A, Yasukawa S, Zen Y et al. Comparison of a 22-gauge Franseentip needle with a 20-gauge forward-bevel needle for the diagnosis of type 1 autoimmune pancreatitis: a prospective, randomized, controlled, multicenter study (COMPAS study). Gastrointest Endosc 2020; 91: 373-381 